\title{
No effect of vitamin D supplementation on circulating glucose, insulin or homeostasis model of insulin resistance (HOMA-IR) in adults aged $20-40$ years and $\geq 64$ years
}

\author{
S. Muldowney ${ }^{1}$, T. R. Hill ${ }^{1}$, A. Lucey ${ }^{1}$, K. Seamans ${ }^{1}$, J. M. W. Wallace ${ }^{3}$, G. Horigan ${ }^{3}$, M. S. Barnes ${ }^{3}$, \\ K. D. Cashman ${ }^{1,2}$ and M. Kiely ${ }^{1}$ \\ ${ }^{1}$ Department of Food \& Nutritional Sciences and ${ }^{2}$ Department of Medicine, University College Cork, Cork, Republic of \\ Ireland and ${ }^{3}$ Northern Ireland Centre for Food and Health, University of Ulster, Coleraine BT52 1SA, UK
}

Observational studies have shown that vitamin D insufficiency is associated with increased risk of type 2 diabetes, insulin resistance and higher plasma glucose concentrations ${ }^{(1)}$; however, intervention trials to date have been limited ${ }^{(2,3)}$. The effect of vitamin D supplementation $(0,5,10$ and $15 \mu \mathrm{g}$ cholecalciferol/d) on serum glucose and insulin concentrations and the HOMA-IR was investigated in two randomised placebo-controlled double-blind 22-week intervention studies in men and women aged 20-40 years $\left(n\right.$ 215; during winter 2006- $\left.7^{(4)}\right)$ and $\geq 64$ years $\left(n 215\right.$; during winter $2007-8^{(5)}$ ) in Cork and Coleraine. Fasting serum levels of glucose were measured by an enzymic colorimetric assay and fasting serum insulin, intact parathyroid hormone (iPTH), and 25-hydroxyvitamin D (25(OH)D) were measured by ELISA at baseline and end point.

\begin{tabular}{|c|c|c|c|c|c|c|c|c|c|c|c|c|c|c|c|c|c|}
\hline \multirow[t]{3}{*}{$\begin{array}{l}\text { Supplement } \\
\text { dose ... }\end{array}$} & \multicolumn{4}{|c|}{$\begin{array}{l}\text { Group } 1 \\
\text { Placebo }\end{array}$} & \multicolumn{4}{|c|}{$\begin{array}{c}\text { Group } 2 \\
5 \mu \mathrm{g} / \mathrm{d}\end{array}$} & \multicolumn{4}{|c|}{$\begin{array}{c}\text { Group } 3 \\
10 \mu \mathrm{g} / \mathrm{d} \\
\end{array}$} & \multicolumn{4}{|c|}{$\begin{array}{c}\text { Group } 4 \\
15 \mu \mathrm{g} / \mathrm{d} \\
\end{array}$} & \multirow[b]{3}{*}{$P$} \\
\hline & \multicolumn{2}{|c|}{ Baseline } & \multicolumn{2}{|c|}{ End point } & \multicolumn{2}{|c|}{ Baseline } & \multicolumn{2}{|c|}{ End point } & \multicolumn{2}{|c|}{ Baseline } & \multicolumn{2}{|c|}{ End point } & \multicolumn{2}{|c|}{ Baseline } & \multicolumn{2}{|c|}{ End point } & \\
\hline & Mean & $\overline{\mathrm{SD}}$ & Mean & $\overline{\mathrm{SD}}$ & Mean & $\overline{\mathrm{SD}}$ & Mean & SD & Mean & $\overline{\mathrm{SD}}$ & Mean & SD & Mean & $\overline{\mathrm{SD}}$ & Mean & $\overline{\mathrm{SD}}$ & \\
\hline $20-40$ years & \multicolumn{4}{|c|}{$(n 56)$} & \multicolumn{4}{|c|}{$(n 50)$} & \multicolumn{4}{|c|}{ (n 57) } & \multicolumn{4}{|c|}{$(n$ 52) } & \\
\hline Glucose $(\mathrm{mmol} / \mathrm{l})$ & 5.04 & 0.7 & 5.04 & 0.7 & 4.90 & 0.8 & 5.18 & 1.4 & 4.90 & 0.5 & 4.98 & 0.6 & 4.92 & 0.8 & 4.99 & 0.7 & 0.653 \\
\hline Insulin $(\mu \mathrm{U} / \mathrm{l})$ & 14.2 & 8 & 15.2 & 8 & 15.1 & 9 & 15.2 & 7 & 15.0 & 9 & 14.1 & 6 & 15.9 & 10 & 14.4 & 6 & 0.275 \\
\hline HOMA-IR & 3.3 & 2 & 3.5 & 2 & 3.4 & 2 & 3.5 & 2 & 3.4 & 2 & 3.2 & 2 & 3.6 & 3 & 3.2 & 2 & 0.350 \\
\hline $25(\mathrm{OH}) \mathrm{D}(\mathrm{mmol} / \mathrm{l})$ & 76.8 & 33 & $41.8^{\mathrm{a}}$ & 18 & 71.3 & 27 & $53.4^{\mathrm{b}}$ & 15 & 77.6 & 33 & $62.1^{\mathrm{c}}$ & 22 & 79.7 & 30 & $72.4^{\mathrm{d}}$ & 21 & $<0.0001^{(4)}$ \\
\hline$\geq 64$ years & \multicolumn{4}{|c|}{$(n 56)$} & \multicolumn{4}{|c|}{$(n$ 51) } & \multicolumn{4}{|c|}{$(n$ 57) } & \multicolumn{4}{|c|}{$(n$ 51) } & \\
\hline Glucose $(\mathrm{mmol} / \mathrm{l})$ & 4.90 & 1.1 & 4.90 & 1.2 & 5.20 & 1.1 & 5.03 & 0.8 & 5.23 & 1.2 & 5.04 & 0.9 & 5.11 & 0.8 & 5.12 & 1.0 & 0.264 \\
\hline Insulin $(\mu \mathrm{U} / \mathrm{l})$ & 9.1 & 8 & 11.9 & 11 & 12.6 & 17 & 11.7 & 15 & 11.1 & 10 & 12.6 & 14 & 10.6 & 7 & 11.3 & 10 & 0.926 \\
\hline HOMA-IR & 2.1 & 3 & 2.8 & 3 & 2.9 & 4 & 2.7 & 3.4 & 2.6 & 3 & 2.9 & 3 & 2.4 & 2 & 2.6 & 2 & 0.958 \\
\hline $25(\mathrm{OH}) \mathrm{D}(\mathrm{mmol} / \mathrm{l})$ & 59.3 & 23 & $43.1^{\mathrm{a}}$ & 17 & 57.2 & 23 & $58.0^{\mathrm{b}}$ & 16 & 59.3 & 26 & $70.6^{\mathrm{c}}$ & 18 & 53.7 & 18 & $76.2^{\mathrm{c}}$ & 21 & $<0.0001^{(5)}$ \\
\hline
\end{tabular}

a,b,c,d Means with unlike subscript letters were significantly different across the four treatment groups $(P<0.05)$.

ANOVA showed no baseline differences in serum levels of glucose, insulin, HOMA-IR or 25(OH)D between the four treatment groups. In adults aged 20-40 years linear regression analysis showed BMI as the main predictor of baseline serum glucose $(\beta 0.344, P<0.0001)$, insulin $(\beta$ 0.357, $P<0.0001)$ and HOMA-IR $(\beta$ 0.0.382, $P<0.0001)$. Male gender was predictive of higher serum glucose concentration $(\beta-0.184, P<0.005)$, while increasing age was predictive of lower insulin concentration $(\beta-0.151, P<0.025)$. In adults aged $\geq 64$ years BMI and iPTH were the main predictors of baseline serum glucose $(\beta 0.184, P<0.008 ; \beta-0.204, P<0.003$ respectively), whereas the main predictor for insulin and HOMA-IR was BMI ( $\beta$ 0.404, $P<0.0001 ; \beta 0.409, P<0.0001$ respectively). In both age-groups ANCOVA revealed no significant effect of the intervention on glucose, insulin or HOMA-IR concentrations across the four treatment groups, adjusting for centre, age, gender, BMI, vitamin D and calcium intakes, iPTH and 25(OH)D at baseline.

In conclusion, vitamin D supplementation had no effect on fasting serum glucose and insulin concentrations or the HOMA-IR in apparently-healthy adults aged $20-40$ and $\geq 64$ years.

We wish to acknowledge the UK Food Standards Agency and the Irish Department of Agriculture, Food \& Fisheries through the Food Institutional Research Measure for their support.

1. Pittas AG, Lau J, Hu FB et al. (2007) J Clin Endocrinol Metab 92, 2017-2029.

2. Nagpal J, Pande JN \& Bhartia A. (2009) Diabet Med 26, 19-27.

3. Pittas AG, Harris SS, Stark PC et al. (2007) Diabetes Care 30, 980-986.

4. Cashman KD, Hill TR, Lucey AJ et al. (2008) Am J Clin Nutr 88, 1535-1542.

5. Cashman KD, Wallace JMW, Horigan G et al. (2009) Am J Clin Nutr 89, 1-9. 